\begin{tabular}{|c|c|c|c|c|c|c|c|c|c|c|c|c|c|c|c|c|c|c|c|c|}
\hline \multirow{2}{*}{$T$} & \multirow{2}{*}{\multicolumn{4}{|c|}{${ }_{\alpha}^{1913 \text { Mai } 3 I}$}} & \multirow{2}{*}{\multicolumn{4}{|c|}{ Juni 5}} & \multirow{2}{*}{\multicolumn{4}{|c|}{ Juni 10}} & \multicolumn{4}{|c|}{ Juni 15} & \multicolumn{4}{|c|}{ Juni 20} \\
\hline & & & & & & & & & & & & & & & 8 & & & & 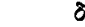 & \\
\hline$-20^{d}$ & & $7 \mathrm{~m}$ & $25^{\circ}$ & $2^{\prime}$ & & $144^{m} \cdot 7$ & +28 & ${ }^{\circ}$ I $3^{\prime}$ & & $3^{2 \mathrm{~m}} \cdot 9$ & $+3 I^{\circ}$ & $2 \mathbf{I}^{\prime}$ & & $2^{m} \cdot 5$ & $+34^{\circ}$ & $23^{\prime}$ & & ${ }^{\mathrm{h}} 3^{\mathrm{m}} \cdot 7$ & $+37^{\circ}$ & ${ }^{\circ}$ I 8 ' \\
\hline--16 & o & 41.8 & 2 & 5 & 0 & $5 \mathrm{I} .4$ & +0 & 23 & I & I. 3 & +3 & $\circ$ & I & 11.7 & +5 & 48 & I & 22.4 & +8 & 46 \\
\hline I. 2 & 23 & 5 I.6 & 24 & 7 & 23 & 57.9 & -23 & 6 & 0 & 4. I & -22 & 3 & 0 & 10.0 & $-2 I$ & 0 & 0 & 15.7 & -19 & 53 \\
\hline-8 & 23 & 10.5 & 39 & 10 & 23 & 14.6 & 39 & 6 & 23 & 18.3 & 39 & 4 & 23 & 2 I. 6 & 39 & 5 & 23 & $24 \cdot 3$ & 39 & 9 \\
\hline-7 & 23 & 0.9 & 42 & 3 & 23 & 4.6 & 42 & 7 & 23 & 7.8 & 42 & 15 & 23 & 10.4 & 42 & 25 & 23 & I 2.6 & 42 & $3^{8}$ \\
\hline-6 & 22 & $5^{1 \cdot 5}$ & 44 & $3^{8}$ & 22 & 54.7 & 44 & 50 & 22 & $57 \cdot 4$ & 45 & 5 & 22 & $59 \cdot 5$ & 45 & 22 & 23 & I. I & 45 & 43 \\
\hline-5 & 22 & 42.2 & 46 & 59 & 22 & 44.9 & 47 & 16 & 22 & 47.2 & 47 & 36 & 22 & 48.8 & 48 & o & 22 & 49.8 & 48 & 26 \\
\hline-4 & 22 & 33.0 & 49 & 5 & 22 & $35 \cdot 3$ & 49 & 27 & 22 & 37.1 & 49 & $5^{2}$ & 22 & $3^{8.2}$ & 50 & 20 & 22 & $3^{8.6}$ & $5^{\circ}$ & $5^{\circ}$ \\
\hline-3 & 22 & 23.9 & $5^{I}$ & o & 22 & 25.8 & $5 I$ & 26 & 22 & $27 \cdot I$ & 51 & 54 & 22 & 27.8 & $5^{2}$ & 25 & 22 & 27.6 & $5^{2}$ & $5^{8}$ \\
\hline-2 & 22 & 15.0 & $5^{2}$ & 44 & 22 & 16.4 & 53 & I 3 & 22 & I $7 \cdot 3$ & 5.3 & 43 & 22 & I $7 \cdot 5$ & 54 & 16 & 22 & I 6.8 & 54 & $5 \mathrm{I}$ \\
\hline$-I$ & 22 & 6.0 & 54 & I 8 & 22 & $7 \cdot I$ & 54 & 49 & 22 & $7 \cdot 5$ & 55 & $2 I$ & 22 & 7.2 & 55 & $5^{6}$ & 22 & 6.0 & $5^{6}$ & 32 \\
\hline$T_{0}$ & $2 \mathrm{I}$ & 57.1 & 55 & 44 & 21 & 57.8 & 56 & I 6 & 2 I & 57.8 & $5^{6}$ & 49 & $2 \mathrm{I}$ & 57.0 & 57 & 25 & $2 \mathrm{I}$ & 55.4 & $5^{8}$ & I \\
\hline+1 & 21 & 48.3 & 57 & I & $2 I$ & 48.6 & 57 & 34 & $2 \mathrm{I}$ & 48.2 & 58 & 9 & $2 \mathrm{I}$ & 47.0 & $5^{8}$ & 44 & $2 I$ & 44.9 & 59 & $2 I$ \\
\hline+2 & 2 I & 39.6 & $5^{8}$ & I 2 & $2 \mathrm{I}$ & 39.5 & $5^{8}$ & $4^{6}$ & 21 & $3^{8.7}$ & 59 & 20 & $2 \mathrm{I}$ & $37 \cdot 3$ & 59 & $5^{6}$ & $2 \mathrm{I}$ & 34.5 & 60 & 32 \\
\hline+3 & $2 \mathrm{I}$ & 30.9 & 59 & I 6 & $2 \mathrm{I}$ & 30.5 & 59 & $5^{\circ}$ & 2 I & $29 \cdot 3$ & 60 & 26 & 21 & 27.2 & $6 I$ & I & $2 \mathrm{I}$ & 24.2 & $6 \mathrm{I}$ & 36 \\
\hline+4 & $2 I$ & 22.3 & 60 & I 5 & $2 \mathrm{I}$ & 21.5 & 60 & 49 & $2 \mathrm{I}$ & I 9.9 & $6 I$ & 24 & 2 I & 17.5 & $6 \mathrm{I}$ & 59 & $2 \mathrm{I}$ & I 4. I & 62 & 32 \\
\hline+5 & $2 \mathrm{I}$ & 13.8 & $6 I$ & 8 & 21 & I 2.7 & $6 I$ & 42 & 21 & 10.7 & 62 & 17 & 21 & 7.8 & 62 & 50 & 2 I & 4. I & 63 & 23 \\
\hline 6 & $2 \mathrm{I}$ & $5 \cdot 4$ & $6 I$ & $5^{6}$ & $2 I$ & 3.9 & 62 & 30 & $2 \mathrm{I}$ & I. 5 & 63 & 4 & 20 & 58.3 & 63 & 37 & 20 & 54.2 & 64 & 9 \\
\hline+7 & 20 & 57.0 & 62 & 40 & 20 & 55.2 & 63 & I 3 & 20 & $5^{2.5}$ & 63 & 46 & 20 & 48.9 & 64 & I 9 & 20 & 44.5 & 64 & 49 \\
\hline+8 & 20 & 48.8 & 63 & 20 & 20 & 46.6 & 63 & 53 & 20 & 4.3 .6 & 64 & 25 & 20 & 39.7 & 64 & $5^{6}$ & 20 & 34.9 & 65 & 25 \\
\hline+12 & 20 & 16.8 & -65 & 27 & 20 & I 3.5 & -65 & $5^{6}$ & 20 & 9.3 & -66 & 24 & 20 & $4 \cdot 3$ & -66 & $5^{\circ}$ & 19 & $5^{8.6}$ & -67 & I 3 \\
\hline
\end{tabular}

\begin{tabular}{|c|c|c|c|c|c|c|c|c|c|c|c|c|c|c|c|c|}
\hline \multirow{2}{*}{$T$} & \multicolumn{2}{|c|}{ April It } & \multicolumn{2}{|c|}{ April 2I } & \multicolumn{2}{|c|}{ Mai I } & \multicolumn{2}{|c|}{ Mai I I } & \multicolumn{2}{|c|}{ Mai 2I } & \multicolumn{2}{|c|}{ Mai 3 I } & \multicolumn{2}{|c|}{ Juni Io } & \multicolumn{2}{|c|}{ Juni 20} \\
\hline & $\log \Delta$ & $H$ & $\operatorname{og} 4$ & $H$ & $\log 1$ & $H$ & $\log \Delta$ & $H$ & $\log \Delta$ & $H$ & $\log \Delta$ & $H$ & $\log \Delta$ & $H$ & $\log 1$ & $H$ \\
\hline$--24^{\mathrm{d}}$ & 0.3508 & $7^{m} \cdot 3$ & 3420 & $7^{\mathrm{m}} \cdot 2$ & & & & & & & & & & & & \\
\hline-20 & 0.4073 & 8.2 & $0.3^{8} 57$ & 8.0 & $0.3^{6} 38$ & $7^{m} \cdot 7$ & 0.3424 & $7^{m} \cdot 5$ & 0.3223 & $7^{m} \cdot 3$ & 0.3049 & $7^{m} 1$ & 0.29 II & $7 \div 0$ & $0.28 \mathrm{II}$ & $6^{m} \cdot 9$ \\
\hline-16 & 0.4657 & 9.1 & 0.4392 & 8.9 & 0.4102 & 8.6 & 0.3788 & 8.4 & $0.345^{2}$ & 8. $\mathrm{I}$ & 0.3095 & 7.8 & 0.2720 & $7 \cdot 5$ & 0.2335 & 7.2 \\
\hline- I 2 & 0.5235 & 9.9 & 0.4968 & 9.7 & 0.4675 & 9.5 & 0.4354 & 9.2 & 0.4001 & 9.0 & 0.36 I 5 & 8.7 & 0.3189 & 8.4 & 0.2724 & 8. I \\
\hline-8 & $0.577 \mathrm{I}$ & I 0.6 & 0.5524 & I 0.4 & $0.5^{2} 5^{6}$ & 10.2 & 0.4963 & 10.0 & 0.4649 & 9.8 & 0.43 I I & $9 \cdot 5$ & 0.3949 & $9 \cdot 3$ & $0.35^{6} 3$ & 9.0 \\
\hline & 0.6022 & 10.9 & 0.5785 & 10.7 & $0.55^{29}$ & 10.5 & $0.5^{2} 5^{8}$ & 10.3 & 0.4967 & IO.I & 0.4659 & 9.9 & $0.433^{6}$ & $9 \cdot 7$ & 0.3999 & 9.4 \\
\hline & $0.625^{6}$ & I 1.2 & 0.6034 & I I I.O & 0.5794 & 10.8 & 0.5540 & 10.6 & 0.5272 & 10.5 & 0.4993 & 10.3 & 0.4705 & 10.0 & 0.4412 & 9.8 \\
\hline & 0.6480 & I I.4 & 0.6268 & I 1.2 & 0.6043 & I I. I & 0.5807 & 10.9 & $0.55^{6 \mathrm{I}}$ & 10.8 & 0.5308 & 16.6 & 0.5053 & 10.4 & 0.4797 & 10.2 \\
\hline$T_{0}$ & 0.6690 & I 1.6 & 0.6490 & I I. 5 & 0.6279 & I I.4 & 0.6059 & I I.2 & $0.5^{8} 3.3$ & I 1.0 & 0.5604 & 10.9 & 0.5376 & 10.7 & 0.5153 & I 0.6 \\
\hline+2 & $0.689 \mathrm{I}$ & I 1.9 & 0.6702 & I 1.7 & 0.6503 & $1 \times .6$ & 0.6298 & I I. 5 & 0.6089 & $\mathrm{Ix} \cdot 3$ & 0.5881 & I 1.2 & 0.5676 & I I I & $0.548 \mathrm{I}$ & 10.9 \\
\hline & $0.708 \mathrm{I}$ & I $2 . \mathrm{I}$ & $0.690 \mathrm{I}$ & $\begin{array}{l}11.9 \\
\end{array}$ & 0.67 I 4 & I 1.8 & 0.6522 & I I. 7 & $0.633^{\circ}$ & I 1.5 & 0.6 I 40 & I I . 4 & $0.595^{6}$ & I I 3 & 0.5782 & I 1.2 \\
\hline+6 & $0.726 \mathrm{I}$ & 12.3 & 0.7090 & I 2.1 & $0.69 \times 3$ & 12.0 & 0.6735 & 11.9 & $0.655^{6}$ & I I .8 & 0.6382 & I 1.7 & 0.6216 & I 1.5 & 0.6062 & I I. 4 \\
\hline+8 & 0.7432 & I 2.5 & 0.7270 & $12 \cdot 3$ & 0.7102 & 12.2 & 0.69 .35 & I 2.1 & 0.6769 & I 2.0 & 0.6609 & I I .9 & $0.645^{8}$ & I 1.8 & $0.632 \mathrm{I}$ & I 1.7 \\
\hline+ & & & & & & & & & 0.7 I 60 & I 2.4 & 0.7023 & 12.3 & 0.6898 & 12.2 & 0.6786 & I 2.1 \\
\hline
\end{tabular}

Die Helligkeit ist nach der Formel $H=5^{\mathrm{m}} \mathrm{o}+5^{\log (r d)}$ berechnet.

St. Petersburg, 19 I 3 März 7/20.

M. Viljev.

\title{
Beobachtungen über die Trübung der Atmosphäre.
}

$\mathrm{Da}$ nach meinen Beobachtungen in Biesdorf bei Berlin der Grad der Trübung sich ständig änderte, habe ich zum eventuellen Vergleich mit anderen Orten versucht, ihre Stärke einen Monat lang für jeden Abend zahlenmäßig zu bestimmen. Die im folgenden benutzte Skala wurde so angesetzt, daß bei $\circ, 3,6$, ro Skaleneinbeiten der Reibe nach noch Sterne $5^{\mathrm{m}}$, $4^{\mathrm{m}} \cdot 0,3^{\mathrm{m}} \cdot 0,2^{\mathrm{m}} \cdot \mathrm{o}$ in $60^{\circ}$ Höhe mit freiem Auge am Osthimmel zu sehen waren; eine normale, gute Nacht würde also etwa mit - I zu bezeichnen sein. An den ausgelassenen Tagen war der Himmel entweder bewölkt oder die Beobachtung zu ungenau (Juli 19,20 ). Die Zeit ist etwa $\mathrm{II}^{\mathrm{h}} \mathrm{M} . \mathrm{E} . \mathrm{Z}$.

Berlin, I 9 I 2 Nov. 30.
1912 Trüb. 1912 Trub.| 1912 \begin{tabular}{lll|lll|ll} 
Juli & 6 & 4 & Juli & I 3 & 2
\end{tabular}

\begin{tabular}{rr|rl|l}
7 & 5 & 14 & 3 & 22 \\
9 & 7 & 15 & 4 & $(1$ \\
10 & 10 & 16 & 3 & 24 \\
11 & 4 & 17 & 4 & 25 \\
12 & 1 & 18 & 5 & 26
\end{tabular}

Trüb. 1912 Trib. $3 ?$ Juli 27 ? $\left.{ }^{2}\right)$ (9 3 (9) 30 ? (I $\left.I^{\mathrm{h}}\right)^{2}$ Aug. 3 ro $5^{\text {?2) }} \quad 4 \quad 5 ?^{\text {? })}$ $\left.?^{4}\right)$

1) Dunst. 2) Ci-st. $\left.{ }^{3}\right)$ Sehr dicker Ci-st.

Übrigens scheint die Wärmeausstrahlung durch die Trübung nicht behindert worden zu sein; z. B. betrug Aug. 3 bei sehr starker Trübung die Luftemperatur um $18^{\mathrm{h}}$ nur $10^{\circ} .3 \mathrm{C}$.

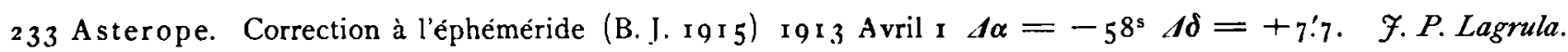

